\title{
RNA-Seq-Based Analysis of the Physiologic Cold Shock- Induced Changes in Moraxella catarrhalis Gene Expression
}

\author{
Violeta Spaniol ${ }^{1 *}$, Stefan Wyder ${ }^{2}$, Christoph Aebi ${ }^{1,3}$ \\ 1 Institute for Infectious Diseases, University of Bern, Bern, Switzerland, 2 Department of Clinical Research, University of Bern, Bern, Switzerland, 3 Department of \\ Pediatrics, University of Bern, Inselspital, Bern, Switzerland
}

\begin{abstract}
Background: Moraxella catarrhalis, a major nasopharyngeal pathogen of the human respiratory tract, is exposed to rapid downshifts of environmental temperature when humans breathe cold air. The prevalence of pharyngeal colonization and respiratory tract infections caused by $M$. catarrhalis is greatest in winter. We investigated how $M$. catarrhalis uses the physiologic exposure to cold air to regulate pivotal survival systems that may contribute to $M$. catarrhalis virulence.

Results: In this study we used the RNA-seq techniques to quantitatively catalogue the transcriptome of $M$. catarrhalis exposed to a $26^{\circ} \mathrm{C}$ cold shock or to continuous growth at $37^{\circ} \mathrm{C}$. Validation of RNA-seq data using quantitative RT-PCR analysis demonstrated the RNA-seq results to be highly reliable. We observed that a $26^{\circ} \mathrm{C}$ cold shock induces the expression of genes that in other bacteria have been related to virulence a strong induction was observed for genes involved in high affinity phosphate transport and iron acquisition, indicating that $M$. catarrhalis makes a better use of both phosphate and iron resources after exposure to cold shock. We detected the induction of genes involved in nitrogen metabolism, as well as several outer membrane proteins, including ompA, $m 35$-like porin and multidrug efflux pump ( $a c r A B$ ) indicating that $M$. catarrhalis remodels its membrane components in response to downshift of temperature. Furthermore, we demonstrate that a $26^{\circ} \mathrm{C}$ cold shock enhances the induction of genes encoding the type IV pili that are essential for natural transformation, and increases the genetic competence of $M$. catarrhalis, which may facilitate the rapid spread and acquisition of novel virulence-associated genes.
\end{abstract}

Conclusion: Cold shock at a physiologically relevant temperature of $26^{\circ} \mathrm{C}$ induces in $M$. catarrhalis a complex of adaptive mechanisms that could convey novel pathogenic functions and may contribute to enhanced colonization and virulence.

Citation: Spaniol V, Wyder S, Aebi C (2013) RNA-Seq-Based Analysis of the Physiologic Cold Shock-Induced Changes in Moraxella catarrhalis Gene Expression. PLoS ONE 8(7): e68298. doi:10.1371/journal.pone.0068298

Editor: Yung-Fu Chang, Cornell University, United States of America

Received March 5, 2013; Accepted May 28, 2013; Published July 2, 2013

Copyright: (c) 2013 Spaniol et al. This is an open-access article distributed under the terms of the Creative Commons Attribution License, which permits unrestricted use, distribution, and reproduction in any medium, provided the original author and source are credited.

Funding: This work was supported by the Swiss National Science Foundation (SNF) grants 3100A0-102246 and 3100A0-116053 (to CA). The funders had no role in study design, data collection and analysis, decision to publish, or preparation of the manuscript.

Competing Interests: The authors have declared that no competing interests exist.

* E-mail: violeta.spaniol@ifik.unibe.ch

\section{Introduction}

Moraxella catarrhalis colonizes the mucosal surface of the human nasopharynx and is a major cause of acute otitis media in children and of exacerbations of chronic obstructive pulmonary disease in adults [1], [2]. The routine use of pneumococcal conjugate vaccines has altered nasopharyngeal colonization patterns and caused an increased prevalence of colonization and infection by M. catarrhalis [3], [4]. Moreover, clinical studies revealed that the prevalence of pharyngeal colonization and respiratory tract infections caused by $M$. catarrhalis displays seasonal variation and increases in winter [5], [6], [7], [8]. Viral infections occurring during the cold season pave the way for subsequent bacterial secondary infection by $\mathrm{T}$-cell mediated release of interferon- $\gamma$, which inhibits bacterial phagocytosis by macrophages [9], and increases the expression of adhesion receptors on epithelial cells such as carcinoembryonic antigen-related cell adhesion molecule 1 (CEACAM1) [10]. In addition, the human nasopharyngeal flora is repeatedly exposed to rapid downshifts of environmental temper- ature. Breathing cold air (e.g., $-1{ }^{\circ} \mathrm{C}$ at $10-20 \mathrm{l} / \mathrm{min}$ ) reduces the nasopharyngeal temperature from $34^{\circ} \mathrm{C}$ at room temperature to about $26^{\circ} \mathrm{C}$ within several minutes and for extended periods of time [11]. Such rapid downshift of temperature induces adaptive events in the residential upper respiratory tract flora that may contribute to the transition from asymptomatic colonization to bacterial secondary infection.

Our previous studies demonstrated that a $26^{\circ} \mathrm{C}$ cold shock upregulates the expression of important virulence traits, such as adherence to epithelial cells, iron acquisition, complement resistance and immune evasion [12], [13]. An increased expression of the UspAl adhesin on the surface of $M$. catarrhalis at $26^{\circ} \mathrm{C}$ leads to an increased adherence to upper respiratory tract epithelial cells in vitro [12], [14]. Exposure of M. catarrhalis to $26^{\circ} \mathrm{C}$ also increases the outer membrane protein (OMP)-mediated release of the proinflammatory cytokine IL-8 in pharyngeal epithelial cells [12] and enhances the expression of genes involved in the acquisition of transferrin and lactoferrin [13]. In contrast, cold shock decreases 
the expression of hemagglutinin (hag/mid), a major lower respiratory tract adhesin, which mediates $\mathrm{B}$ cell response, and reduces immunoglobulin D-binding on the surface of $M$. catarrhalis. Furthermore, cold shock reduces the expression of porin M35, which may affect the resistance to aminopenicillins [15]. Thus, the physiologic cold shock appears to be an important contributor to the seasonal peak in $M$. catarrhalis infections and could be clinically relevant during the cold season by temporarily increasing the organism's virulence.

In the present study, we performed a comprehensive transcriptome analysis in $M$. catarrhalis exposed to $37^{\circ} \mathrm{C}$ or to a $26^{\circ} \mathrm{C}$ cold shock using the Illumina ${ }^{\circledR}$ RNA-seq technique. We were able to define global changes in gene expression in response to cold shock. We observed that a $26^{\circ} \mathrm{C}$ cold shock induces the expression of genes that in other bacteria have been related to virulence. These data provided broader and deeper insights into the transcriptional regulation in $M$. catarrhalis after a $26^{\circ} \mathrm{C}$ cold shock and also provide a rich basis for further investigations.

\section{Materials and Methods}

\section{Bacterial Strains and Culture Conditions}

$M$. catarrhalis strain $\mathrm{O} 35 \mathrm{E}$ and the clinical isolate 415 have been described elsewhere [13], [14]. Bacteria were cultured at $37^{\circ} \mathrm{C}$ and $200 \mathrm{rpm}$ in brain heart infusion (BHI) broth (Difco) or on BHI agar plates in an atmosphere containing $5 \% \mathrm{CO}_{2}$. Cold shock experiments were performed as described [14]. Bacteria were grown overnight at $37^{\circ} \mathrm{C}$, resuspended in fresh medium and grown to mid-logarithmic phase (optical density at $600 \mathrm{~nm}\left[\mathrm{OD}_{600}\right]$ of 0.3 . Subsequently, bacteria were exposed to $26^{\circ} \mathrm{C}$ or $37^{\circ} \mathrm{C}$, respectively, for 3 hours.

\section{RNA Preparation and Illumina RNA-seq}

$M$. catarhalis cultures were fixed with 2 volumes of RNA protect (Qiagen) and harvested. RNA was isolated using the RNeasy kit (Qiagen) as described elsewhere [14]. Residual DNA in the samples was removed using Dnase I. The integrity of the RNA was analyzed using an Agilent bioanalyzer (Agilent technologies). The Ribo-Zero rRNA removal kit (Gram-negative bacteria, Epicentre) was used to remove the $23 \mathrm{~S}$ and $16 \mathrm{~S}$ rRNA from the total RNA samples. Library construction and sequencing were performed by the Genomics Core Facility of the Department of Clinical Research, University of Bern using TruSeq RNA sample preparation v2 guide (Illumina). Sequencing raw data generated in this study are available at GEO, accession number GSE46256 (http://www.ncbi.nlm.nih.gov/geo/query/acc. cgi? $\cdot a c c=$ GSE46256).

\section{RNA-seq Data Analysis}

The quality analysis of RNA-seq data was carried out using the FastQC application (http://www.bioinformatics.babraham.ac.uk/ projects/fastqc/). The $M$. catarrhalis $\mathrm{O} 35 \mathrm{E}$ genome sequence as well as open reading frame (ORF) positions and annotations were obtained using the RefSeq and PATRIC database [16] (http:// patricbrc.vbi.vt.edu/portal/portal/patric/Home and http:// brcdownloads.vbi.vt.edu/patric2/genomes/

Moraxella_catarrhalis_O35E). Reads were mapped to the $M$. catarrhalis O35E RefSeq genome sequence using the BWA algorithm [17]. The summarization of counts was performed using the BEDTools software [18], and normalization and the differential expression analysis was performed using the variance analysis package DEseq [19] using $\mathrm{R}$ version 2.8.0. The total read count was determined for each gene by combining data from three biological replicate sequencing runs. $P$-values were calculated and adjusted for multiple testing using the false discovery rate (FDR) controlling procedure [20]. Functional classification of the $M$. catarrhalis $\mathrm{O} 35 \mathrm{E}$ genes was projected from the $M$. catarrhalis $\mathrm{RH} 4$ genome [21] by identifying the orthologous proteins between these strains using the best reciprocal blast hit approach with protein sequences. For all but seven proteins from the O35E strain an 1:1 orthologous protein could be identified in the RH4 strain. The seven O35E proteins were equally similar to multiple (2 or 3 ) different RH4 proteins. Enrichment analysis was performed using the functional categories with a custom python skript (Fisher's exact test) correcting for multiple testing using the FDR.http:// www.nature.com/nature/journal/v486/n7402/full/nature1 1234 . html - refl2.

\section{Quantitative Reverse Transcriptase PCR (qRT-PCR) Assays}

RNA was isolated as described in the previous section. As templates for this assay we used the one set of RNA samples that was used for the synthesis of first Illumina library and two additional pairs of samples that were independently obtained. The reverse transcription step was carried out using the Superscript II cDNA synthesis kit (Invitrogen) according to the manufacturer's instructions. Oligonucleotide primer pairs (Table S1) were designed for use in qRT-PCR with either PrimerExpress software (Applied Biosystems) or Primer 3 [22]. To assess for DNA contamination, RNA samples were also run without reverse transcriptase. The constitutively expressed $16 \mathrm{~S}$ ribosomal RNA gene was used as an internal control for relative quantification. Reactions for qRT-PCR were completed using Power SYBR green PCR Master Mix (Applied Biosystems) with a two-step reaction protocol consisting of 40 cycles of $94^{\circ} \mathrm{C}$ for $30 \mathrm{~s}$ and $60^{\circ} \mathrm{C}$ for $1 \mathrm{~min}$, followed by a dissociation phase for quality control. The $25-\mu \mathrm{l}$ qPGR mixtures contained $0.2 \mu \mathrm{M}$ specific primers, Power SYBR green (Applied Biosystems) (2x), and $2 \mu \mathrm{l}$ of cDNA (10 ng/ $\mu \mathrm{l})$. Relative quantification of gene expression was performed using the comparative threshold method. The ratios obtained after normalization were expressed as folds of change compared with samples isolated from bacteria exposed to $37^{\circ} \mathrm{C}$.

\section{Natural Transformation}

The quantitative transformation assay was performed as described elsewhere [23]. Briefly, M. catarrhalis cells exposed to $37^{\circ} \mathrm{C}$ or to a $26^{\circ} \mathrm{C}$ were adjusted to an $\mathrm{OD}_{600 \mathrm{~nm}}$ of 0.2 , glycerol was added to a final concentration of $20 \%$ and $200 \mu$ l aliquots of these naturally competent cells were stored at $-70^{\circ} \mathrm{C}$ until use. Aliquots $(100 \mu \mathrm{l})$ were spread on BHI agar plates and air-dried. Thirty $\mu$ l of solution containing $100 \mathrm{ng}$ of purified linear DNA $(M$. catarrhalis uspA1::kan) was spotted onto the lawn in duplicate for each condition, followed by incubation at $26^{\circ} \mathrm{C}$ or $37^{\circ} \mathrm{C}, 5 \% \mathrm{CO}_{2}$. After $5 \mathrm{~h}$, bacteria were harvested and resuspended in $1 \mathrm{ml}$ of $\mathrm{BHI}$ broth. Serial dilutions were prepared and spread on BHI agar plates for total viable cell counts, or on BHI agar plates containing kanamycin at $20 \mathrm{mg} / \mathrm{l}$. After $24 \mathrm{~h}$ of incubation at $37^{\circ} \mathrm{C}$ in $5 \%$ $\mathrm{CO}_{2}$, the number of CFUs per experiment was determined and transformation frequencies were calculated. All experiments were performed in independent triplicates.

\section{Antimicrobial Peptide Susceptibility Assay}

Antimicrobial peptide susceptibility assay was performed as described elsewhere [24]. Bacteria were exposed either to $26^{\circ} \mathrm{C}$ or $37^{\circ} \mathrm{C}$ for $3 \mathrm{~h}$, adjusted to approximately $5 \times 10^{5} \mathrm{CFU} / \mathrm{ml}$ in $100 \mathrm{mM} \mathrm{NaCl}, 2 \% \mathrm{BHI}$ and $10 \mathrm{mM}$ PBS (pH 7). Aliquots $(5 \mu \mathrm{l})$ of this suspension were mixed in 1.5-ml microcentrifuge tubes with various concentrations of polymyxin B (Sigma). In all cases the 
final volume was $30 \mu \mathrm{l}$. After $1 \mathrm{~h}$ of incubation at the bacterial growth temperature, the contents of the tubes were plated onto BHI agar. Colony counts were determined, and results were expressed as percentages of the colony count of bacteria not exposed to the antibacterial agent.

\section{Statistical Analysis}

Data were expressed as mean \pm 1 standard deviation (SD). Differences between groups were analyzed by one-way analysis of variance with a Bonferroni correction using Prism software (version 5.01; GraphPad). $P<0.05$ was defined as statistically significant.

\section{Results and Discussion}

\section{Sequencing of the $M$. catarrhalis Transcriptome after Exposure to $26^{\circ} \mathrm{C}$ and $37^{\circ} \mathrm{C}$}

Whole genome mRNA sequencing was used to monitor the global changes in gene expression of $M$. catarrhatis strain $\mathrm{O} 35 \mathrm{E}$ exposed to a physiological $26^{\circ} \mathrm{C}$ cold shock relative to continuous growth at $37^{\circ} \mathrm{C}$. In order to determine the cold shock stimulon, RNA-seq was performed on three biological replicates of $M$. catarrhalis growth at different temperatures using the Illumina HiSeq 2000 system. Since 16S and 23S rRNA were anticipated to be the most abundant RNA species, these were depleted prior to sequencing by oligonucleotide hybridisation-mediated capture. A total of 183 million single-end reads (100 bp) was obtained of which 47'900'062 reads mapped uniquely to the $M$. catarrhalis O35E strain genome. Between 3.2 and 14.5 million reads for each sample aligned to non-rRNA regions of the $M$. catarrhalis genome representing approximately $25 \%$ of all reads (Table S2), which illustrates the suitability of RNA-seq for bacterial transcriptomic studies. When expression data for each biological replicate were plotted against each other, the normalized counts were highly reproducible (Figure S1). RNA expression levels of $M$. catarrhalis replicates upon exposure to $26^{\circ} \mathrm{C}$ and $37^{\circ} \mathrm{C}$ were highly similar $\left(\mathrm{R}^{2}>0.97\right.$ and $\mathrm{R}^{2}>0.99$, respectively). Genes with differential expression between $26^{\circ} \mathrm{C}$ and $37^{\circ} \mathrm{C}$ were identified using the DEseq [19] package (Table S3). Using a FDR cut-off of $5 \%$ a total of 831 genes were significantly differentially expressed greater than 1.5-fold, including 429 up-regulated genes and 402 downregulated genes at $26^{\circ} \mathrm{C}$ (Table S4). Differential expression was defined according to $P$ value for each gene by the comparison of normalized counts (log2 scale, average of 3 replicates) between $26^{\circ} \mathrm{C}$ and $37^{\circ} \mathrm{C}$ (Figure 1). The magnitude distribution of the significantly differently expressed genes was illustrated by MA plot analysis showing individual gene responses plotted as $\log 2$ foldchange versus mean expression (Figure S2). Next we analyzed whether cold shock-regulated gene transcripts were enriched in specific biological processes (Figure 2A). Among the differentially expressed genes we identified several statistically enriched functional categories (Figure 2B and Table S5). The following categories were enriched among gene transcripts induced upon a cold-shock of $26^{\circ} \mathrm{C}$ : "Transcription" and "Transport and binding proteins". Functional categories enriched among genes repressed at $26^{\circ} \mathrm{C}$ included: "Protein fate", "Protein folding and stabilization", "Purines, pyrimidines, nucleosides, and nucleotides", "Glycolysis/gluconeogenesis", "Fatty acid and phospholipids biosynthesis" and "Molybdopterin biosynthesis".

\section{Validation of Differentially Regulated Genes by qRT-PCR}

Several genes found to be differentially expressed by RNA-seq have been already identified in our previous studies [13], [14], [15]. For example, a $26^{\circ} \mathrm{C}$ cold shock induced expression of genes encoding a major adhesin UspAl (uspA1), genes involved in transferrin and lactoferrin acquisition $(t b p A / B, \quad l b p A / B)$ and reduced the expression of genes encoding a M35-porin (m35) and hemagglutinin $(\mathrm{hag} / \mathrm{mid})$. To further corroborate the RNA-seq data, several differentially expressed genes were selected for secondary confirmation using quantitative RT-PCR analysis. As shown in Figure 3, qRT-PGR experiments confirmed that the expression of these genes was regulated by temperature. The fold changes detected for each gene were similar to the fold changes observed by RNA-seq. A similar expression profile was observed when $M$. catarrhalis clinical isolate 415 was exposed to $26^{\circ} \mathrm{C}$ and $37^{\circ} \mathrm{C}$, with the exception that no transcription of the gene encoding the m35-like protein was found (data not shown).

\section{Differentially Expressed Genes Involved in Transcription and Replication}

Cold causes formation and stabilization of secondary structures in RNA, which will interfere with efficient ribosomal binding, elongation and translation termination [25]. Bacteria have evolved several established strategies to counteract the deleterious influence of cold-induced mRNA structuring. One of them is based on the cooperative binding of RNA chaperones (CspA family) to nascent mRNA transcripts for preventing the formation of mRNA secondary structures. This process leads to preferential translation of cold-inducible mRNA [25]. The CspA family in E. coli comprises several homologous proteins that are cold inducible [26]. Two cold shock proteins, CspA and cold shock-like protein, were predicted by genome analysis of $M$. catarrhalis [21]. Our data demonstrate that cold shock strongly enhanced (4.8-fold) the transcription of cold shock-like protein, whereas the expression of the gene encoding cold shock protein CspA was only moderately (1.4-fold) increased.http://www.sciencedirect.com/science/ article/pii/S1286457905000079 - bib34\#bib34 The ATP-dependent RNA helicase-encoding genes $(r h l B)$, whose transcription was enhanced (1.9-fold) after temperature downshift, are known to be involved in the resolving of the cold-induced structuring of mRNA [27].

Cold shock causes a severe impairment of the translation machinery, leading to a block in translation initiation and decoupling of transcription to translation [28]. Several genes (infB, rho, nus $A B$ ) involved in modulating of translation have been identified to be cold shock inducible, indicating that activation of translation is accomplished by enhanced expression of translation factors and components specifically associated with the ribosome. We found that numerous genes involved in the transcription process were significantly induced after exposure to cold shock (Figure 2B and Table S5) including several transcription factors (dksA, rho, nusA/B, nadR) and DNA-dependent RNA polymerases $(r p o A, r p o C)$.

Low temperature affects the conformation, flexibility and topology of DNA, which in turn have a deleterious influence on DNA replication [29]. In our study, several genes involved in DNA replication, recombination, and repair $(\operatorname{dnaA} / E, \mathrm{rec} \mathcal{N} / G, \operatorname{top} A)$ as well as genes involved in restriction/modification were identified to be up-regulated upon cold shock.

Among the down-regulated genes upon shift from $37^{\circ} \mathrm{C}$ to $26^{\circ} \mathrm{C}$ were genes encoding major heat shock proteins GroES, GroEL and DnaK. This suggests that under conditions of a $26^{\circ} \mathrm{C}$ cold shock, bacteria no longer require elevated expression of these genes.

The analysis of potential transcriptional regulators within the $M$. catarrhalis genome revealed, that cold shock increased the expression of several regulatory DNA-binding proteins including cold shock-like protein (4.8-fold), nitrite-sensitive transcriptional 


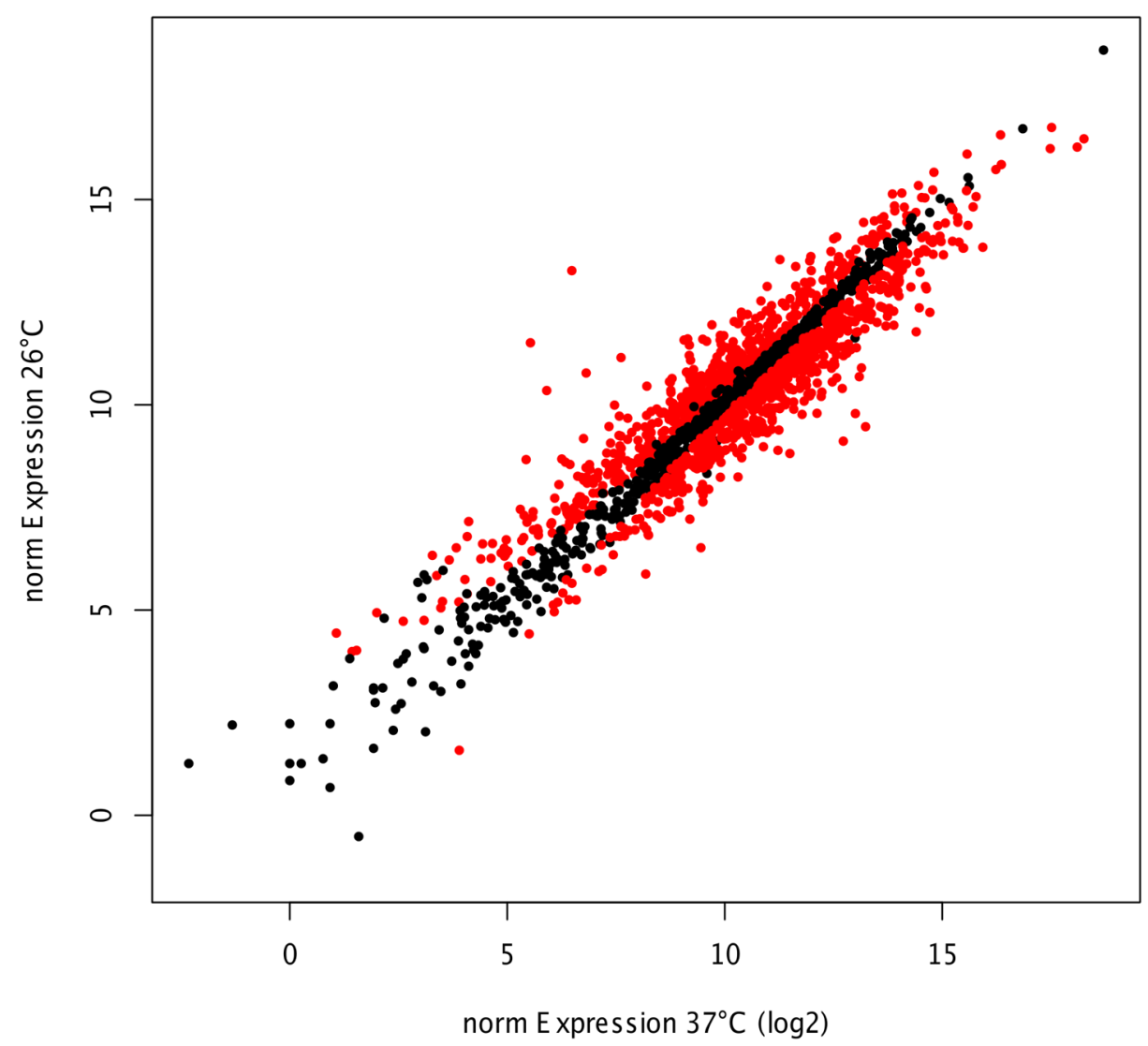

Figure 1. Differentially expressed genes following cold shock. The scatterplot shows normalized counts for $M$. catarrhalis either grown at $26^{\circ} \mathrm{C}$ or $37^{\circ} \mathrm{C}$ (log2 scale, mean of three biological replicates). Each dot represents an individual gene. Genes showing significant differential expression in the RNA-seq data are highlighted in red, genes that are not regulated by temperature are highlighted in black. The genes are highlighted according their adjusted p-value (False Discovery Rate). doi:10.1371/journal.pone.0068298.g001

repressor NsrR (4.8-fold), LysR (2.2-fold) and the AsnC (2.2-fold) family transcriptional regulators. The two-component system phosphate regulon response regulator PhoB (2-fold) and sensor histidine kinase PhoR (2.1-fold) were also induced following cold shock. However, two TetR family transcriptional regulators (1.6 and 1.8-fold), two-component system nitrate/nitrite response regulator NarL and sensor histidine kinase NarX were upregulated (2.3- and 1.7 -fold, respectively) at $37^{\circ} \mathrm{C}$.

\section{Differentially Expressed Genes Involved in Carbon und Energy Metabolism}

Generally, genes involved in energy metabolism particularly in glycolysis/gluconeogenesis and electron transport were expressed at a greater level after exposure of M.catarrhalis to $37^{\circ} \mathrm{C}$ (Figure 2A and 2B, Table S5). M. catarrhalis possesses an incomplete glycolytic pathway, indicating the inability to utilize exogenous carbohydrates [30]. All of the enzymes of the gluconeogenic pathway are present, suggesting that carbohydrate intermediates can be synthesized [21], [31]. Gluconeogenesis uses phosphoenolpyruvate (PEP) as a starting substrate, which can be generated from tricarboxylic acid (TCA) cycle intermediates. The expression of numerous glycolytic enzymes $(f b p, g a p A, g p m I$, eno, $p p s A$, aceE), Dlactate dehydrogenase $(d l d)$ and L-lactate dehydrogenase $(l l d D)$ was at a greater level at $37^{\circ} \mathrm{C}$. Acetate kinase $(a c k A)$, required for conversion of acetate into acetyl-CoA, was also upregulated at $37^{\circ} \mathrm{C}$. In contrast, triose-phosphate isomerase (tpiA) that catalyses the reversible conversion of glyceraldehyde-3-phosphate and dihydroxyacetone and glucose-6-phosphate isomerase $(p g i)$ were upregulated after exposure to $26^{\circ} \mathrm{C}$.

The TCA cycle is supplied with acetyl coenzyme A (acetyl-CoA) via degradation of fatty acids and acetate assimilation. The enzymes of the TCA cycle were induced after exposure to cold shock conditions. Genes encoding succinate dehydrogenase (sdhA), aconitate hydratase $(a c n B)$ and enzyme of the glyoxylate bypass $($ ace $B)$, that are required for utilization of acetyl-coenzyme $\mathrm{A}(\mathrm{CoA})$ during gluconeogenesis, were upregulated after temperature shift to $26^{\circ} \mathrm{C}$. The malic enzyme $($ maeB $)$, which converts malate to the level of pyruvate, which can enter the TCA cycle, was upregulated after exposure to $37^{\circ} \mathrm{C}$. Studies on Yersinia pestis showed that a shift from $26^{\circ} \mathrm{C}$ to $37^{\circ} \mathrm{C}$ caused downregulation of numerous glycolytic enzymes $(g a p A, g m p A$, eno), whereas the carbohydrate transport systems (eg. ABC galactose transporter, $m g l B A C$ ) and a full TCA cycle were upregulated at $37^{\circ} \mathrm{C}$ [32]

The majority of genes encoding enzymes involved in aerobic respiration and electron transport was enhanced after continuous exposure of $M$. catarrhalis to $37^{\circ} \mathrm{C}$, including the cytochrome c oxidase subunits (ccoGPS), cytochrome c-type biogenesis protein $(\mathrm{ccm} E)$, cytochrome d ubiquinol oxidase subunits $(c y d A B)$, thioredoxin $(t r x)$ and thioredoxin reductase $(\operatorname{tr} x R)$.

Several genes encoding enzymes involved in aerobic respiration and electron transport were also induced upon cold shock, including the rubredoxin family protein, NADH dehydrogenase subunits $($ nuoEG $)$ and $4 \mathrm{Fe}-4 \mathrm{~S} / 2 \mathrm{Fe}-2 \mathrm{~S}$ ferredoxins. Exposure $M$. catarrhalis to a $26^{\circ} \mathrm{C}$ cold shock thus can induce the energy 
A

A

Amino acid biosynthesisBiosynthesis of cofactors, prosthetic groups, and carriersCellular processes Central intermediary metabolism DNA metabolism Energy metabolism Fatty acid and phospholipid metabolism Mobile and extrachromosomal element functions Protein synthesis

Purines, pyrimidines, nucleosides, and nucleotides

Regulatory functions Rignal transduction Transport and binding proteins Unknown function-

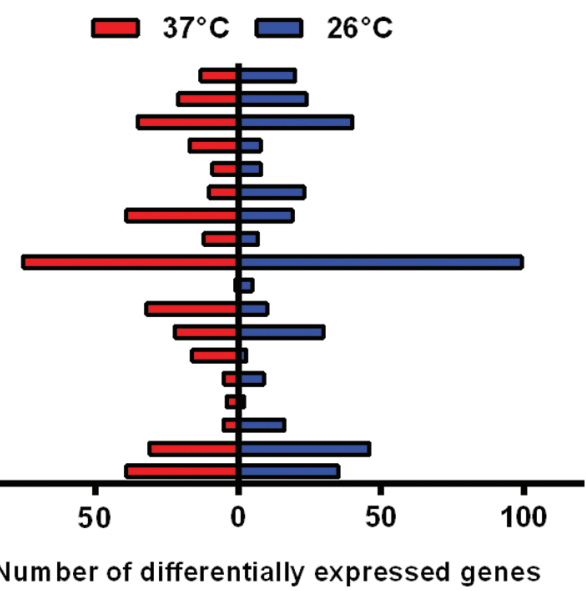

B

Transcription
Transport and binding protein
Protein fate
Protein folding and stabilization
Purines, pyrimidines,
nucleosides, and nucleotides
Glycolysis/gluconeogenesis
Fatty acid and phospholipid
biosynthesis
Molybdopterin biosynthesis

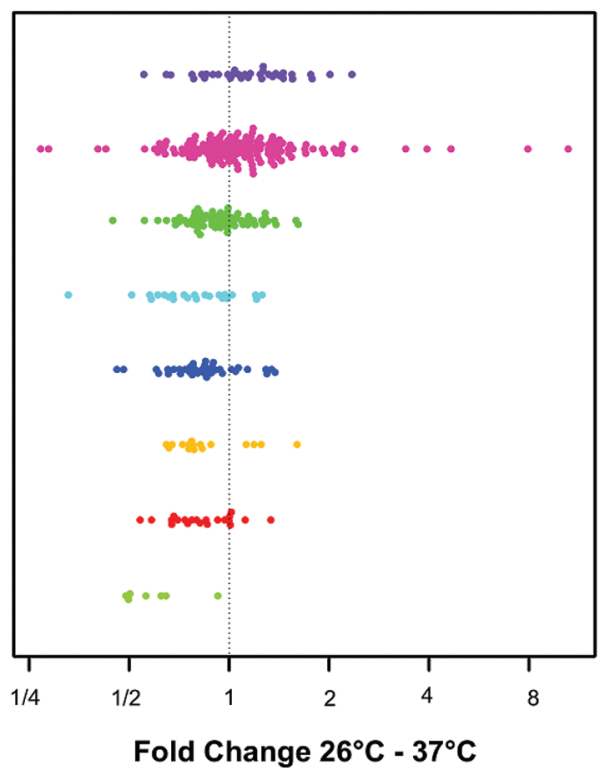

Figure 2. Functional categories of M.catarrhalis genes induced or repressed following cold shock. A. Differentially expressed genes (Table S4) were broadly categorized according to their biological function. Each bar represents the actual number of genes. Blue bars indicate number of genes that are upregulated at $26^{\circ} \mathrm{C}$, and red bars represent number of genes that are downregulated at $26^{\circ} \mathrm{C}$. B. The sets of up- or downregulated genes (based on DESeq analysis) were tested for enrichment in specific functional categories. Average log2 fold-changes of individual genes are shown for categories with adjusted $p$-values $<5 \%$.

doi:10.1371/journal.pone.0068298.g002

generation, which ensures ATP-dependent responses such as removing RNA secondary structures with ATP-dependent RNA helicases and increased degradation of fatty acids during cold adaptation.

\section{Differentially Expressed Genes Involved in Lipid Metabolism}

Shift to $26^{\circ} \mathrm{C}$ caused in $M$. catarrhalis the induction of several genes encoding enzymes ( $f a d E, f a d f, f a d D / f a d K, p g p A)$ involved in fatty acid degradation that subsequently supplies the TCA cycle with acetyl coenzyme A (acetyl-CoA). However, cold shock reduced the expression of several genes ( $f a b B, f a b H$, accABCD) encoding enzymes or regulators involved in fatty acid biosynthesis. Furthermore, outer membrane protein E (ompE), a putative FadL homolog and long-chain-fatty-acid transporter is induced (2.3-fold) after exposure to $37^{\circ} \mathrm{C}$. The transcription of $r$. pestis genes $(\mathrm{fabF} /$ $f a b \mathcal{F}, f a b H, f a b G, f a b E$, and $f a d R$ ), encoding enzymes or regulators involved in fatty acid biosynthesis, were induced by a $10^{\circ} \mathrm{C}$ cold shock, indicating the increase of fatty acid biosynthesis during $\Upsilon$. pestis cold adaptation [33].

\section{Differentially Expressed Genes Involved in cell Envelope Biosynthesis}

Lipooligosaccharide (LOS) is a major component of the outer leaflet of the outer membrane of gram-negative bacteria. In this study, the mRNA level of several genes $(l p x B, l p x X, k d t A$ and $\operatorname{lgt} 6)$ involved in LOS biosynthesis was enhanced by cold shock. Similarly, $Y$. pestis comprises several homologous proteins (lp $x A$, $l p \times B, k d t A)$ that are cold inducible [32], [33]. In contrast, we found that the expression of genes encoding UDP-3-O-N-acetylglucosamine deacetylase $(l p x C)$ and dodecanoyltransferase $(l p x L)$ were enhanced after exposure to $37^{\circ} \mathrm{C}$. $\mathrm{LpxB}$ is a key enzyme 


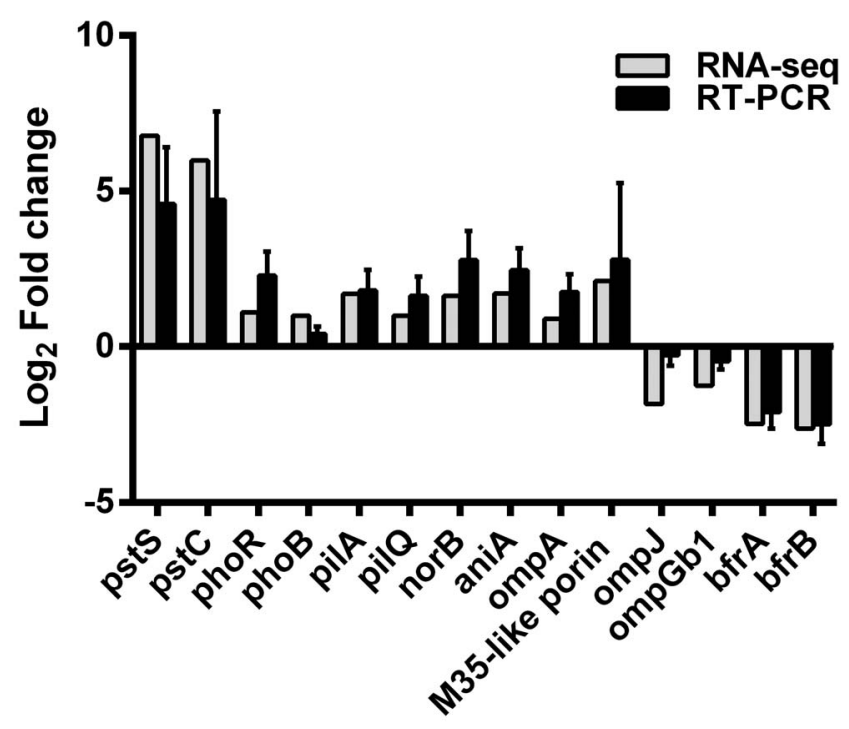

Figure 3. qRT-PCR validation of differentially expressed genes. Fold changes of the expression levels of genes pstS encoding phosphate $A B C$ transporter, periplasmic phosphate-binding protein PstS; pstC encoding phosphate transport system permease protein PstC; phoR encoding two-component system phosphate regulon sensor histidine kinase PhoR; phoB encoding two-component system phosphate regulon response regulator PhoB; pilA encoding type IV pilin PilA; pilQ encoding type IV pilus secretin PilQ; nor $B$ encoding nitric oxide reductase NorB; aniA encoding nitrite reductase AniA/Msp78; ompA encoding OmpA family protein; $m 35$-like porin encoding M35-like porin protein, ompJ encoding outer membrane protein J, ompG $1 b$ encoding outer membrane protein $\mathrm{G} 1 \mathrm{~b} ;$; $f r A$ and $b f r B$ encoding bacterioferritins were compared to those detected by RNA-seq. The log2 of the fold difference in gene expression between $26^{\circ} \mathrm{C}$ and $37^{\circ} \mathrm{C}$ temperatures as determined by qRT-PCR (black columns) is plotted adjacent to the results obtained in RNA-seq analysis (grey columns). The results of qRTPCR are expressed as means \pm 1 SD of three separate experiments performed in duplicate.

doi:10.1371/journal.pone.0068298.g003

participating in lipid A biosynthesis. The Kdo transferase gene $k d t A$ catalyzes the addition of $\mathrm{Kdo}$ residues to the lipid $\mathrm{A}$ precursor. The $l p x L$ and $l p x X$ genes encode late acyltransferases that are responsible for the incorporation of secondary acyl chains into lipid A. The expression levels of the acyltransferases are temperature regulated, and the lipid A acylation status affects the expression of Yersinia enterocolitica virulence factors [34]. Decreased acylation allows the bacteria to evade detection by the host innate immune system. Induction of the innate immune system by modifying the tetraacylated lipid A structure by overexpression of the late acyltransferase LpxL in $\Upsilon$. pestis results in a complete loss of virulence [35]. Previous studies demonstrated that $Y$. enterocolitica is more susceptible to polymyxin B, a model of antimicrobial peptides of the innate immune system, when grown at $37^{\circ} \mathrm{C}$ than at $22^{\circ} \mathrm{C}$ by interacting with the anionic lipid A moiety of the LPS [24]. Therefore, we hypothesized that temperature may also regulate the expression of $M$. catarrhalis lipid A modifications linked to antimicrobial peptides resistance. However, we found no differences in the susceptibility of $M$. catarrhalis O35E exposed to a $26^{\circ} \mathrm{C}$ cold shock or to continuous growth at $37^{\circ} \mathrm{C}$ to polymyxin $\mathrm{B}$ (data not shown), indicating that a $26^{\circ} \mathrm{C}$ may not be sufficiently low to induce remarkable changes in the LOS structure, or that the reciprocal action of the $M$. catarrhalis $\mathrm{LpxX}$ and $\mathrm{LpxL}$ acyltransferases may compensate this effect.

The peptidoglycan layer is the main target for B-lactam antibiotics that can be degraded by the Bro beta-lactamases produced by $M$. catarrhalis [36]. The transcription of gene encoding the beta-lactamase family protein $(b r o-1)$ involved in resistance was increased (2.5-fold) at $37^{\circ} \mathrm{C}$.

Type IV pili (TFP) have a wide variety of functions, including adhesion to epithelial cells, biofilm formation and motility, and play a crucial role in the initiation of disease by a wide range of human mucosal pathogens [37]. Furthermore, expression of TFP by $M$. catarrhalis is essential for natural competence [38]. This mechanism of DNA transformation is a major contributor to the horizontal exchange of genetic information between naturally competent microorganisms [39]. Analysis of RNA-seq data demonstrated that a $26^{\circ} \mathrm{C}$ cold shock caused in $M$. catarrhalis the induction of genes encoding type IV pilin A (pilA, 3.2-fold), type IV pilus secretin (pilQ 2.0-fold) and several type IV pilus biogenesis proteins (pilBCDT). Moreover, the transcription of genes encoding major competence proteins (comEA, comM) and the DNA protecting protein $(d p r A)$ was strongly induced in $M$. catarrhalis by cold shock. It has been demonstrated, that the type IV A pilinencoding gene, pilA, expression of Burkholderia pseudomallei was dramatically increased following growth at $27^{\circ} \mathrm{C}$ compared to growth at $37^{\circ} \mathrm{C}$ [40]. In contrast, the surface expression of the Enterococcus faecium PilA- and PilB-type pili was regulated in a temperature-dependent manner, as polymerization of two distinct types of pili at the surface only occurred when cells were grown at $37^{\circ} \mathrm{C}$; no pili were observed on cells grown at $21^{\circ} \mathrm{C}$ [41]. Similarly, expression of pil operon was strongly induced after growth of $Y$. pseudotuberculosis at $37^{\circ} \mathrm{C}$ as compared to growth at $28^{\circ} \mathrm{C}$ [42].

Increased methylation state has been shown to enhance the transformation efficiency in Neisseria gonorrhoeae protecting foreign DNA from digestion by restriction enzymes [43]. The expression of gene encoding the type III restriction system methylase was strongly induced at $26^{\circ} \mathrm{C}(5.8$-fold $)$ in comparison to $37^{\circ} \mathrm{C}$ suggesting that cold shock increases the transformation efficiency also by protecting foreign DNA from own restriction modification systems.

Because the transcription of type IV pili was found to be induced after incubation at $26^{\circ} \mathrm{C}$, and the expression of TFP is correlated with natural genetic transformation in many bacterial species, we investigated whether cold shock conditions affect the natural genetic competence of $M$. catarrhalis. The contribution of cold shock to $M$. catarrhalis DNA competence was investigated in a quantitative transformation assay. M. catarrhalis strain O35E was exposed to $26^{\circ} \mathrm{C}$ or $37^{\circ} \mathrm{C}$ and evaluated for their differences in the natural transformation frequencies. The DNA transformation experiments revealed that $M$. catarrhalis following a $26^{\circ} \mathrm{C}$ cold shock show greater (more than 4-fold) natural transformation frequencies than bacteria exposed to $37^{\circ} \mathrm{C}$ (Figure 4). The natural genetic competence of M.catarrhalis was also increased (3-fold) after cold shock in bacteria during planctonic growth (data not shown). Similarly, cold shock increases (2.1-fold) the competence of M. catarrhalis clinical isolate 415 (data not shown).

Analysis of RNA-seq data demonstrated that a $26^{\circ} \mathrm{C}$ cold shock caused in $M$. catarrhalis a significant induction of numerous genes encoding transport and binding proteins (Figure 2B and Table S5). Examples are OmpA family protein (1.8-fold), M35-like porin (4.3fold) as well as systems involved in transport of amino acids, peptides and amines like oligopeptide $\mathrm{ABC}$ transport system $(o p p B D F$ ), arginine $\mathrm{ABC}$ transporter (artQ), amino acid $\mathrm{ABC}$ transporter $(o c c M)$, systems involved in anions transport like phosphate $\mathrm{ABC}$ transporter system (pstACS), nitrate $\mathrm{ABC}$ transporter $(n r t A B C D)$, systems involved in transport of cations and iron carrying compounds like transferrin and lactoferrin binding proteins $(t b p A B, \operatorname{lbp} A B)$, acetate permease ActP (cation/acetate symporter), chelated iron $\mathrm{ABC}$ transporter $(a f e B C D)$ and motA/ 


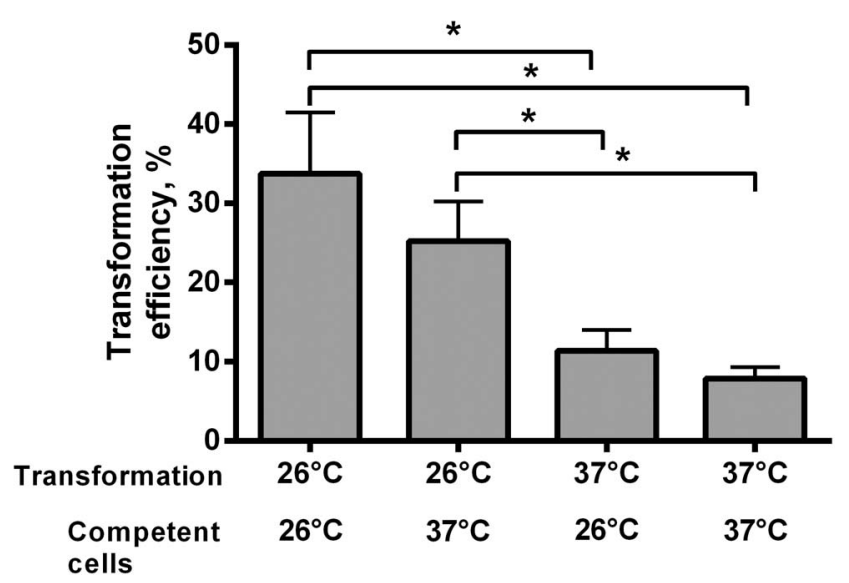

Figure 4. Influence of temperature on natural transformation of M. catarrhalis. M. catarrhalis strain $\mathrm{O} 35 \mathrm{E}$, growth at $26^{\circ} \mathrm{C}$ or $37^{\circ} \mathrm{C}$, was transformed with $100 \mathrm{ng}$ of purified linear DNA (M. catarrhalis uspA1::kan) and incubated at $26^{\circ} \mathrm{C}$ or $37^{\circ} \mathrm{C}$ for $5 \mathrm{~h}$. Transformation frequencies are expressed as the transformant CFU per total CFU and represent the average of three independent assays performed in duplicates. ${ }^{*}, P<0.05$, one-way analysis of variance. doi:10.1371/journal.pone.0068298.g004

TolQ/ExbB proton channel. It has been shown that in E.coli human body temperature increases amino acid, carbohydrate and iron uptake and utilization gene expression compared to growth at $23^{\circ} \mathrm{C}$ [44]. A major outer membrane protein A (OmpA) of Acinetobacter baumannï plays a partial role in biofilm formation and is absolutely required for attachment to epithelial cells [45]. Several lines of evidence have shown that the OmpA of E. coli and Klebsiella pneumoniae can protect bacteria from bactericidal effects [46], [47].

\section{Differentially Expressed Genes Involved in Protein Fate}

Many genes encoding proteins associated with protein fate were found to be induced after exposure to $37^{\circ} \mathrm{C}$ (Figure 2B and Table S5). Especially the genes encoding proteins involved in protein folding and stabilization displayed increased expression at $37^{\circ} \mathrm{C}$. Two protein secretion systems (Tat and Sec) that transport proteins from the cytosol across the inner membrane to the periplasm were found in the $M$. catarrhalis genome [21]. Genes encoding the Tat translocation proteins $(\operatorname{tat} A B)$ were induced at $37^{\circ} \mathrm{C}$. In contrast, a $26^{\circ} \mathrm{C}$ cold shock caused in $M$. catarrhalis the induction of genes encoding the Sec protein secretion system $(\sec G Y)$ as well as genes encoding components of the resistance-nodulation-division (RND) multidrug efflux systems (acrAB, oprM) [48].

\section{Differentially Expressed Genes Involved in Nitrogen Metabolism}

The denitrification pathway of $M$. catarrhalis provides an alternative mechanism to generate energy under lower oxygen tension and contributes to biofilm formation and virulence [31]. The genes of the denitrification pathway encoding the nitrite reductase (aniA, 3.3-fold), nitric oxide reductase (nor $B, 3.1$-fold) as well as the nitrate $\mathrm{ABC}$ transporter complex (nrtABCD) were strongly upregulated after exposure to a $26^{\circ} \mathrm{C}$ cold shock. Interestingly, under conditions of cold shock the transcription of the repressor gene, $n s r R$, is also increased (4.8-fold), a mechanism that may prevent the uncontrolled overexpression of the denitrfication pathway genes. The upregulation of the denitrification genes after exposure of $M$. catarrhalis to $26^{\circ} \mathrm{C}$ may indicate that there is limited oxygen availability in this environment. Glutamine synthetase $(g \ln A)$ involved in nitrogen assimilation was also upregulated upon shift to cold shock conditions. In contrast, the genes encoding the two-component system nitrate/nitrite response regulator NarL and nitrate/nitrite transporters (narK1/K2) were down-regulated after exposure to $26^{\circ} \mathrm{C}$. Similarly, many genes of $\Upsilon$. pestis involved in nitrogen assimilation were strongly upregulated upon shift to $26^{\circ} \mathrm{C}$ [32]. The nasopharyngeal mucosa is rich in nitric oxide (NO)-producing cells, including macrophages and epithelial cells, and local concentrations of NO have been shown to reach concentrations which are likely to be greatly in excess of that required to produce toxic effects in most bacteria [49]. The inreased ability to reduce nitric oxide after exposure to $26^{\circ} \mathrm{C}$ may also provide $M$. catarrhalis with some level of protection against macrophage-generated nitric oxide. The ability of $\mathcal{N}$. meningitidis to survive in nasopharyngeal mucosal tissue has been shown to be enhanced by expression of nitric oxide detoxification systems [50].

\section{Differentially Expressed Genes Involved in Iron Acquisition}

Our previous findings established that a $26^{\circ} \mathrm{C}$ cold shock upregulates the expression of genes involved in transferrin and lactoferrin acquisition, and enhances binding of these proteins on the surface of M. catarrhalis [13]. Analysis of RNA-seq data demonstrated that expression levels of genes involved in iron transport and utilization were significantly increased at $26^{\circ} \mathrm{C}$ in comparison to $37^{\circ} \mathrm{C}$. The transcription of genes encoding transferrin binding proteins $(t b p A / B, 1.7$ - and 2.4-fold respectively) and lactoferrin binding proteins $(l b p A / B, 4.4-$ and 2-fold, respectively) was upregulated after exposure to $26^{\circ} \mathrm{C}$. The afe $B C D$ gene cluster, involved in the acquisition of chelated iron, was also upregulated after exposure to $26^{\circ} \mathrm{C}$. In contrast, two bacterioferritins A/B $(b f r A / B)$, involved in intracellular iron storage, were strongly (5.5- and 6.2-fold, respectively) down-regulated at $26^{\circ} \mathrm{C}$. Efficient iron acquisition from lactoferrin is an important virulence factor for pathogenic mucosal bacteria. It has been shown that supplemental lactoferrin can enhance the virulence of meningococcal infection in mice [51]. Increased expression of $M$. catarrhalis lactoferrin and transferrin binding proteins following cold shock would facilitate the binding and acquisition of iron from lactoferrin/transferrin to support growth of bacteria in the mucosal environment.

\section{Differentially Expressed Genes Involved in Phosphate Metabolism}

Expression levels of genes involved in phosphate transport and utilization were significantly increased at $26^{\circ} \mathrm{C}$ in comparison to $37^{\circ} \mathrm{C}$. Examples are phosphate $\mathrm{ABC}$ transporter proteins (pstABCS) involved in the capture of periplasmic inorganic phosphate and its transport into the cytosol, and the PhoBR $(p h o B / R)$, a two-component regulatory system that appears to respond to external phosphate concentrations through interaction with a phosphate transport system and controls gene transcription of the Pho regulon [52]. Noticeably, phosphate ABC transporter periplasmic phosphate-binding protein $\mathrm{PstS}$ and phosphate transport system permease protein $\mathrm{PstC}$ stand out as the top two most upregulated genes in M.catarrhalis exposed to a $26^{\circ} \mathrm{C}$ cold shock, respectively. PstS was upregulated 110 -fold, while PstC was upregulated 63-fold. Recent studies have shown that the Pho regulon plays a role in the virulence of several bacteria. The Pst system is found to be involved in modifications of lipid A and fatty acid composition [53]. An inactivating pst mutation in E. coli affects multiple virulence attributes; it reduces resistance to the bactericidal effect of serum, to acidity and to cationic antimicrobial peptides [54]. 


\section{Differentially Expressed Genes Involved in Sulfur Metabolism}

Transcription of genes from the sulfur assimilation pathway (cys $D H N I$ ) were upregulated after exposure of $M$. catarrhalis to cold shock. Sulfur metabolic pathways are essential for survival and the expression of virulence in many pathogenic bacteria, including Mycobacterium tuberculosis [55]. The genes of the sulfate assimilation pathway are consistently upregulated in $M$. tuberculosis by diverse environmental cues, including nutrient starvation, hypoxia, oxidative stress, and cell wall stress [56].

\section{Differentially Expressed Genes Involved in Oxidative Stress}

The superoxide dismutase-catalase system is able to counteract the effect of oxidative stress by catalyzing the conversion of superoxide to water and oxygen [57]. The expression of genes involved in oxidative stress response including superoxide dismutase A ( $\operatorname{sod} A, 1.9$-fold), catalase (katA, 2-fold), alkyl hydroperoxide reductase $(\operatorname{ah} p C / F, 4.3$-and 3.6-fold, respectively), peroxiredoxin family protein/glutaredoxin (2.9-fold) was increased at $37^{\circ} \mathrm{C}$. Activation of these genes suggests that $M$. catarrhalis may be exposed to reactive oxygen species (ROS) under $37^{\circ} \mathrm{C}$ conditions.

\section{Differentially Expressed Genes Involved in Nucleotide Metabolism}

Nucleotides are important substrates for DNA synthesis and DNA repair. Analysis of RNA-seq data demonstrated that exposure of M.catarrhalis to $37^{\circ} \mathrm{C}$ caused significant induction of numerous genes involved in nucleotide metabolism (Figure 2B and Table S5). The majority of genes involved in purine (purCHMN, guaB, $\operatorname{prs} A)$ and pyrimidine (pyrBDE, carA) biosynthesis as well as genes involved in nucleotide and nucleoside interconversions $\mathrm{gmk}$, pyrH, $n d k$ ) were upregulated at $37^{\circ} \mathrm{C}$. It has been shown that de novo nucleotide biosynthesis is critical for survival and growth of $\mathrm{E}$. coli in human serum [58].

Many pathogenic bacteria respond to an increase from environmental temperatures to that of a mammalian body by the expression of genes that promote survival in the mammalian host [59]. Typically, these pathogens occupy at some stage of their life cycle an environmental reservoir such as soil, water, or an arthropod host from which mammalian hosts become infected. Bacterial species known to upregulate virulence gene expression in response to a shift to mammalian body temperature include Yersinia, Borrelia, Shigella, Bordetella, E. coli and Streptococcus [59]. Following a temperature upshift to $37^{\circ} \mathrm{C}, B$. pertussis, $Y$. enterocolitica and $Y$. pseudotuberculosis activate the transcription of early genes that encode the factors required for bacterial adhesion [60], [61]. Similarly, the human body temperature increases in E. coli the expression of genes involved in adhesion as well as in iron and amino acids uptake and utilization [44].

M. catarrhalis, however, does not belong to the environmental microflora, but is an exclusively human mucosal pathogen and commensal. The $M$. catarrhalis cumulative nasopharyngeal colonization rate in young children is high (up to 75\%) [1], [62], suggesting that the major source of transmission of $M$. catarrhalis to infants are adults and other young children. In contrast to other bacteria, a temperature downshift to $26^{\circ} \mathrm{C}$ activates the expression of the uspA1 gene, a major adhesin of $M$. catarrhalis, and increases the adherence of M.catarrhalis to pharyngeal epithelial cells in vitro [12], [14]. Therefore, the relevance to pathogenesis of a highly adherent phenotype when bacteria are exposed to a $26^{\circ} \mathrm{C}$ cold shock merits discussion. In our previous studies we also found that a $26^{\circ} \mathrm{C}$ cold shock increases the proinflammatory effect on epithelial cells by enhanced release of the proinflammatory cytokine IL-8 [12]. Cold shock also affects the expression of genes involved in immune evasion: exposure to $26^{\circ} \mathrm{C}$ decreases the expression of hemagglutinin (Hag), a major adhesin, which mediates B cell responses, and reduces immunoglobulin D-binding on the surface of $M$. catarrhalis. This may reduce the stimulation of $\mathrm{B}$ cells and increase bacterial survival by prevention of endocytosis by these cells [13].

Cold shock-induced changes, which occur in the transcription of several membrane-related genes, demonstrate that $M$. catarrhalis may remodel its membrane components in response to a downshift of temperature. Many of these genes are known as major virulence factors. M. catarrhalis acquires many of the nutrients it requires for growth directly from the host. Under conditions of low temperature in the nasopharynx various nutrients may become limited as the metabolism of host cells slows. M. catarrhalis appears to respond by up-regulating proteins with homology to transporters and binding proteins, which are involved in the transport of nutrients from the host (iron, phosphate, amino acids).

We also found that a $26^{\circ} \mathrm{C}$ cold shock increases the expression of genes involved in the biosynthesis of pili leading to increased natural transformation rates. Competence for natural genetic transformation is a programmed physiologic state, which enables bacteria to take up and process exogenous DNA [63]. A rapid increase in the prevalence of $\beta$-lactamase-producing $M$. catarrhalis strains has been observed during in the last 30 years such that more than $95 \%$ of clinical isolates now appear to be resistant to one or more $\beta$-lactams [64]. Increased transcription of genes encoded the TFP and greater natural genetic transformation rates, which were observed after exposure of $M$. catarrhalis to a $26^{\circ} \mathrm{C}$ cold shock, may facilitate the rapid spread and acquisition of novel virulence-associated genes in $M$. catarrhalis, as has been shown for the bro $\beta$-lactamase gene, whose sequence suggested that it was acquired by interspecies gene transfer, possibly from a grampositive bacterium [65], [66]. Recent studies reported an increase in resistance to trimethoprim/sulfamethoxazole (cotrimoxazole) of $18.5 \%$ in Taiwan and to $82.5 \%$ in India [67], [68]. Similarly, $80 \%$ of all M. catarrhalis strains tested in the UK and Ireland were resistant to cefaclor, and 5\% to cefuroxime [69]. Furthermore, increased natural transformation following cold shock may also enhance the exchange of virulence properties within the carriage population, leading to new emergent phenotypes with heightened pathogenic potential. A recent study has shown that $M$. catarrhalis can incorporate the UspAl CEACAM1-binding region not only into rare UspAl proteins devoid of a CEACAM-binding region, but also into UspA2, which normally lack this capacity [70]. Conceivably, this could convey novel adhesive functions whilst enhancing immune evasion.

It is conceivable that cold shock could promote the virulence of $M$. catarrhalis by taking advantage of the induction of adherence and several uptake systems that in other bacteria have been related to virulence. Since it has been shown that one stress response might help bacteria to contend with other stress conditions [71], [72], it is possible that cold shock could improve the ability of M. catarrhalis to survive in the host because several pathways of stress responses are activated. Thus, cold shock induces in M. catarrhalis a complex of adaptive mechanisms that could convey novel pathogenic functions and may contribute to enhanced growth and colonization. Clinical studies in children have demonstrated that the density of $M$. catarrhalis in the nasopharynx is positively associated with prolonged respiratory tract symptoms and a greater likelihood of purulent otitis media [73], [74]. Therefore, the physiologic cold shock response may emerge as an important contributor to the seasonal peak in $M$. catarrhalis 
infections in temperate climates. Overall, these studies contribute a wealth of new information on the pathogenetic effect of a $26^{\circ} \mathrm{C}$ cold shock in $M$. catarrhalis.

\section{Supporting Information}

Figure S1 Reproducibility of the RNA-seq replicates. Expression data for each biological replicate $\left(26^{\circ} \mathrm{C}\right.$ vs $\left.37^{\circ} \mathrm{C}, \mathrm{n}=3\right)$ were plotted against each other.

(TIF)

Figure S2 Differentially expressed genes following cold shock. Correlation of fold-change and normalized mean expression (log scale). Genes showing significant differential expression in the RNA-seq data are highlighted in red, genes that are not regulated by temperature are highlighted in black.

(TIF)

\section{Table S1 Oligonucleotides used in this study. (DOC)}

\section{Table S2 Summary of Illumina RNA-seq data.} (DOC)

Table S3 Differentially expressed genes according to DESeq analysis. Genes overexpressed at $26^{\circ} \mathrm{C}$ in comparison with $37^{\circ} \mathrm{C}$ are at the top of this list, genes overexpressed at $37^{\circ} \mathrm{C}$ at the bottom. Genes are sorted according to their p-value.

(XLS)

\section{References}

1. Faden H, Duffy R, Wasielewski R, WolfJ, Krystofik D, et al. (1997) Relationship between nasopharyngeal colonization and the development of otitis media in children. J Infect Dis 175: 1440-5.

2. Palmu A, Herva E, Savolainen H, Karma P, Mäkela PH, et al. (2004) Association of clinical signs and symptoms with bacterial findings in acute otitis media. Clin Infect Dis 38: 234-42.

3. Revai K, McCormick DP, Patel J, Grady JJ, Saeed K, et al. (2006) Effect of pneumococcal conjugate vaccine on nasopharyngeal bacterial colonization during acute otitis media. Pediatrics 117(5): 1823-9.

4. Murphy TF, Parameswaran GI (2009) Moraxella catarrhalis, a human respiratory tract pathogen. Clin Infect Dis 49(1): 124-31.

5. Van Hare GF, Shurin PA (1987) The increasing importance of Branhamella catarrhalis in respiratory infections. Pediatr Infect Dis J 6(1): 92-4.

6. Mbaki N, Rikitomi N, Nagatake T, Matsumoto K (1987) Correlation between Branhamella catarrhalis adherence to oropharyngeal cells and seasonal incidence of lower respiratory tract infections. Tohoku J Exp Med 153(2): 111-21.

7. Sarubbi FA, Myers JW, Williams JJ, Shell CG (1990) Respiratory infections caused by Branhamella catarrhalis. Selected epidemiologic features. Am J Med 88(5A): $9 \mathrm{~S}-14 \mathrm{~S}$.

8. Hendley JO, Hayden FG, Winther B (2005) Weekly point prevalence of Streptococcus pneumoniae, Hemophilus influenzae and Moraxella catarrhalis in the upper airways of normal young children: effect of respiratory illness and season. APMIS 113(3): 213-20.

9. Sun K, Metzger DW (2008) Inhibition of pulmonary antibacterial defense by interferon-gamma during recovery from influenza infection. Nat Med 14(5): 558-64.

10. Griffiths NJ, Bradley CJ, Heyderman RS, Virji M (2007) IFN-gamma amplifies NFkappaB-dependent Neisseria meningitidis invasion of epithelial cells via specific upregulation of CEA-related cell adhesion molecule 1. Cell Microbiol 9(12): 2968-83.

11. Rouadi P, Baroody FM, Abbott D, Naureckas E, Solway J, et al. (1999) A technique to measure the ability of the human nose to warm and humidify air. J Appl Physiol 87(1): 400-6.

12. Spaniol V, Troller R, Aebi C (2009) Physiologic cold shock increases adherence of Moraxella catarrhalis to and secretion of interleukin 8 in human upper respiratory tract epithelial cells. J Infect Dis 200(10): 1593-601.

13. Spaniol V, Troller R, Schaller A, Aebi C (2011) Physiologic cold shock of Moraxella catarrhalis affects the expression of genes involved in the iron acquisition, serum resistance and immune evasion. BMC Microbiol 11: 182.

14. Heiniger N, Troller R, Meier PS, Aebi C (2005) Cold shock response of the UspA1 outer membrane adhesin of Moraxella catarrhalis. Infect Immun 73: 824755 .

15. Jetter M, Spaniol V, Troller R, Aebi C (2010) Down-regulation of porin M35 in Moraxella catarrhalis by aminopenicillins and environmental factors and its potential contribution to the mechanism of resistance to aminopenicillins. J Antimicrob Chemother 65(10): 2089-96.
Table S4 Genes differentially expressed in response to cold shock. Using a FDR cut-off of $5 \%$ a total of 831 genes were significantly differentially expressed greater than 1.5-fold, including 429 up-regulated genes and 402 down-regulated genes at $26^{\circ} \mathrm{C}$.

(XLS)

Table S5 Functional categories of M.catarrhalis genes induced or repressed following cold shock. Frequency lists the number of genes present both in the gene set and in the specific category followed by the total number of reference genes in the category. Enrichment was tested using Fishers's exact test (p-value) and adjusted for multiple testing using FDR (p-value adjusted). Categories with adjusted p-values $<5 \%$ are highlighted in bold. (DOC)

\section{Acknowledgments}

We thank Muriel Fragnière from the Genomics Core Facility, Department of Clinical Research, University of Bern for technical support.

\section{Author Contributions}

Conceived and designed the experiments: VS CA. Performed the experiments: VS. Analyzed the data: VS SW. Contributed reagents/ materials/analysis tools: VS SW CA. Wrote the paper: VS CA.

16. Gillespie JJ, Wattam AR, Cammer SA, Gabbard JL, Shukla MP, et al. (2011) PATRIC: the comprehensive bacterial bioinformatics resource with a focus on human pathogenic species. Infect Immun 79(11): 4286-98.

17. Li H, Durbin R (2009) Fast and accurate short read alignment with BurrowsWheeler transform. Bioinformatics 25(14): 1754-60.

18. Quinlan AR, Hall IM (2010) BEDTools: a flexible suite of utilities for comparing genomic features. Bioinformatics 26(6): 841-2.

19. Anders S, Huber W (2010) Differential expression analysis for sequence count data. Genome Biol 11(10): R106.

20. Benjamini Y, Hochberg Y (1995) Controlling the false discovery rate: a practical and powerful approach to multiple testing. Journal of the Royal Statistical Society Series B: Statistical Methodology 57(1): 289-300.

21. de Vries SP, van Hijum SA, Schueler W, Riesbeck K, Hays JP, et al. (2010) Genome analysis of Moraxella catarrhalis strain RH4, a human respiratory tract pathogen. J Bacteriol 192(14): 3574-83.

22. Rozen S, Skaletsky H (2000) Primer3 on the WWW for general users and for biologist programmers. Methods Mol Biol 132: 365-86.

23. Meier PS, Troller R, Heiniger N, Hays JP, van Belkum A, et al. (2006) Unveiling electrotransformation of Moraxella catarrhalis as a process of natural transformation. FEMS Microbiol Lett 262(1): 72-6.

24. Reines M, Llobet E, Llompart CM, Moranta D, Perez-Gutierrez C, et al. (2012) Molecular basis of Yersinia enterocolitica temperature-dependent resistance to antimicrobial peptides. J Bacteriol 194(12): 3173-88.

25. Weber MH, Marahiel MA (2003) Bacterial cold shock responses. Sci Prog 86(Pt 1-2): 9-75.

26. Phadtare S, Alsina J, Inouye M (1999) Cold-shock response and cold-shock proteins. Curr Opin Microbiol 2(2): 175-80.

27. Jones PG, Mitta M, Kim Y, Jiang W, Inouye M (1996) Cold shock induces a major ribosomal-associated protein that unwinds double-stranded RNA in Escherichia coli. Proc Natl Acad Sci U S A 93(1): 76-80.

28. Farewell A, Neidhardt FG (1998) Effect of temperature on in vivo protein synthetic capacity in Escherichia coli. J Bacteriol 180(17): 4704-10.

29. Lopez-Garcia P, Forterre P (2000) DNA topology and the thermal stress response, a tale from mesophiles and hyperthermophiles. Bioessays 22(8): 73846.

30. Catlin BW (1990) Branhamella catarrhalis: an organism gaining respect as a pathogen. Clin Microbiol Rev 3(4): 293-320.

31. Wang W, Reitzer L, Rasko DA, Pearson MM, Blick RJ, et al. (2007) Metabolic analysis of Moraxella catarrhalis and the effect of selected in vitro growth conditions on global gene expression. Infect Immun 75(10): 4959-71.

32. Motin VL, Georgescu AM, Fitch JP, Gu PP, Nelson DO, et al. (2004) Temporal global changes in gene expression during temperature transition in Yersinia pestis. J Bacteriol 186(18): 6298-305.

33. Han Y, Zhou D, Pang X, Zhang L, Song Y, et al. (2005) DNA microarray analysis of the heat- and cold-shock stimulons in Tersinia pestis. Microbes Infect 7(3): $335-48$. 
34. Perez-Gutierrez C, Llobet E, Llompart CM, Reines M, Bengoechea JA (2010) Role of lipid A acylation in Yersinia enterocolitica virulence. Infect Immun 78(6): 2768-81.

35. Montminy SW, Khan N, McGrath S, Walkowicz MJ, Sharp F, et al. (2006) Virulence factors of Yersinia pestis are overcome by a strong lipopolysaccharide response. Nat Immunol 7(10): 1066-73.

36. Bootsma HJ, van DH, Verhoef J, Fleer A, Mooi FR (1996) Molecular characterization of the BRO beta-lactamase of Moraxella (Branhamella) catarrhalis. Antimicrob Agents Chemother 40(4): 966-72.

37. Luke NR, Jurcisek JA, Bakaletz LO, Campagnari AA (2007) Contribution of Moraxella catarrhalis type IV pili to nasopharyngeal colonization and biofilm formation. Infect Immun 75(12): 5559-64.

38. Luke NR, Howlett AJ, Shao J, Campagnari AA (2004) Expression of type IV pili by Moraxella catarrhalis is essential for natural competence and is affected by iron limitation. Infect Immun 72(11): 6262-70.

39. Aas FE, Wolfgang M, Frye S, Dunham S, Lovold C, et al. (2002) Competence for natural transformation in Neisseria gonorrhoeae: components of DNA binding and uptake linked to type IV pilus expression. Mol Microbiol 46(3): 749-60.

40. Boddey JA, Flegg CP, Day CJ, Beacham IR, Peak IR (2006) Temperatureregulated microcolony formation by Burkholderia pseudomallei requires pilA and enhances association with cultured human cells. Infect Immun 74(9): 5374-81.

41. Hendrickx AP, Bonten MJ, van Luit-Asbroek M, Schapendonk CM, Kragten $\mathrm{AH}$, et al. (2008) Expression of two distinct types of pili by a hospital-acquired Enterococcus faecium isolate. Microbiology 154(Pt 10): 3212-23.

42. Collyn F, Léty MA, Nair S, Escuyer V, Ben Younes A, et al. (2002) Rersinia pseudotuberculosis harbors a type IV pilus gene cluster that contributes to pathogenicity. Infect Immun 70(11): 6196-205.

43. Butler CA, Gotschlich EC (1991) High-frequency mobilization of broad-hostrange plasmids into Neisseria gonorrhoeae requires methylation in the donor. J Bacteriol 173(18): 5793-9.

44. White-Ziegler CA, Malhowski AJ, Young S (2007) Human body temperature $\left(37^{\circ} \mathrm{C}\right)$ increases the expression of iron, carbohydrate, and amino acid utilization genes in Escherichia coli K-12. J Bacteriol 189(15): 5429-40.

45. Gaddy JA, Tomaras AP, Actis LA (2009) The Acinetobacter baumannii 19606 OmpA protein plays a role in biofilm formation on abiotic surfaces and in the interaction of this pathogen with eukaryotic cells. Infect Immun 77(8): 3150-60.

46. Weiser JN, Gotschlich EC (1991) Outer membrane protein A (OmpA) contributes to serum resistance and pathogenicity of Escherichia coli K-1. Infect Immun 59(7): 2252-8.

47. Llobet E, March C, Gimenez P, Bengoechea JA (2009) Klebsiella pneumoniae OmpA confers resistance to antimicrobial peptides. Antimicrob Agents Chemother 53(1): 298-302.

48. Nikaido H (1996) Multidrug efflux pumps of gram-negative bacteria. J Bacteriol 178(20): 5853-9.

49. Lundberg JO, Farkas-Szallasi T, Weitzberg E, Rinder J, Lidholm J, et al. (1995) High nitric oxide production in human paranasal sinuses. Nat Med 1(4): 370-3.

50. Stevanin TM, Moir JW, Read RC (2005) Nitric oxide detoxification systems enhance survival of Neisseria meningitidis in human macrophages and in nasopharyngeal mucosa. Infect Immun 73(6): 3322-9.

51. Schryvers AB, Gonzalez GC (1989) Comparison of the abilities of different protein sources of iron to enhance Neisseria meningitidis infection in mice. Infect Immun 57(8): 2425-9.

52. Wanner BL (1993) Gene regulation by phosphate in enteric bacteria. J Cell Biochem 51(1): 47-54.

53. Lamarche MG, Kim SH, Crépin S, Mourez M, Bertrand N, et al. (2008) Modulation of hexa-acyl pyrophosphate lipid A population under Escherichia coli phosphate (Pho) regulon activation. J Bacteriol 190(15): 5256-64
54. Lamarche MG, Dozois CM, Daigle F, Caza M, Curtiss R 3rd, et al. (2005) Inactivation of the pst system reduces the virulence of an avian pathogenic Escherichia coli O78 strain. Infect Immun 73(7): 4138-45.

55. Bhave DP, Muse WB 3rd, Carroll KS (2007) Drug targets in mycobacterial sulfur metabolism. Infect Disord Drug Targets 7(2): 140-58.

56. Rustad TR, Harrell MI, Liao R, Sherman DR (2008) The enduring hypoxic response of Mycobacterium tuberculosis. PLoS One 3(1): e1502.

57. Farr SB, Kogoma T (1991) Oxidative stress responses in Escherichia coli and Salmonella typhimurium. Microbiol Rev 55(4): 561-85.

58. Samant S, Lee H, Ghassemi M, Chen J, Cook JL, et al. (2008) Nucleotide biosynthesis is critical for growth of bacteria in human blood. PLoS Pathog 4(2): e37.

59. Konkel ME, Tilly K (2000) Temperature-regulated expression of bacterial virulence genes. Microbes Infect 2(2): 157-66.

60. Prugnola A, Arico B, Manetti R, Rappuoli R, Scarlato V (1995) Response of the bvg regulon of Bordetella pertussis to different temperatures and short-term temperature shifts. Microbiology 141 (Pt 10): 2529-34.

61. Straley SC, Perry RD (1995) Environmental modulation of gene expression and pathogenesis in Yersinia. Trends Microbiol 3(8): 310-7.

62. Verhaegh SJ, Snippe ML, Levy F, Verbrugh HA, Jaddoe VW, et al. (2011) Colonization of healthy children by Moraxella catarrhalis is characterized by genotype heterogeneity, virulence gene diversity and co-colonization with Haemophilus influenzae. Microbiology 157(Pt 1): 169-78.

63. Dubnau D (1999) DNA uptake in bacteria. Annu Rev Microbiol 53: 217-44.

64. Khan MA, Northwood JB, Levy F, Verhaegh SJ, Farrell DJ, et al. (2010) bro \{beta\}-lactamase and antibiotic resistances in a global cross-sectional study of Moraxella catarrhalis from children and adults. J Antimicrob Chemother 65(1): 917.

65. Bootsma HJ, van DH, Vauterin P, Verhoef J, Mooi FR (2000) Genesis of BRO beta-lactamase-producing Moraxella catarrhalis: evidence for transformationmediated horizontal transfer. Mol Microbiol 36(1): 93-104.

66. De Vries SP, Bootsma HJ, Hays JP, Hermans PW (2009) Molecular aspects of Moraxella catarrhalis pathogenesis. Microbiol Mol Biol Rev 73(3): 389-406.

67. Hsu SF, Lin YT, Chen TL, Siu LK, Hsueh PR, et al. (2012) Antimicrobial resistance of Moraxella catarrhalis isolates in Taiwan. J Microbiol Immunol Infect 45(2): $134-40$.

68. Gupta N, Arora S, Kundra S (2011) Moraxella catarrhalis as a respiratory pathogen. Indian J Pathol Microbiol 54(4): 769-71.

69. Morrissey I, Maher K, Williams L, Shackcloth J, Felmingham D, et al. (2008) Non-susceptibility trends among Haemophilus influenzae and Moraxella catarrhalis from community-acquired respiratory tract infections in the UK and Ireland, 1999-2007. J Antimicrob Chemother 62 Suppl 2: ii $97-103$.

70. Hill DJ, Whittles C, Virji M (2012) A novel group of Moraxella catarrhalis UspA proteins mediates cellular adhesion via CEACAMs and vitronectin. PLoS One 7(9): e45452.

71. Xu H, Lee HY, Ahn J (2008) Cross-protective effect of acid-adapted Salmonella enterica on resistance to lethal acid and cold stress conditions. Lett Appl Microbiol 47(4): 290-7.

72. Gunasekera TS, Csonka LN, Paliy O (2008) Genome-wide transcriptional responses of Escherichia coli K-12 to continuous osmotic and heat stresses. J Bacteriol 190(10): 3712-20.

73. Kristo A, Uhari M, Kontiokari T, Glumoff V, Kaijalainen T, et al. (2006) Nasal middle meatal specimen bacteriology as a predictor of the course of acute respiratory infection in children. Pediatr Infect Dis J 25(2): 108-12.

74. Smith-Vaughan H, Byun R, Nadkarni M, Jacques NA, Hunter N, et al. (2006) Measuring nasal bacterial load and its association with otitis media. BMC Ear Nose Throat Disord 6: 10 . 